\title{
Fractional metric dimension of metal-organic frameworks
}

https://doi.org/10.1515/mgmc-2021-0012

received November 25, 2020; accepted March 01, 2021

Abstract: Metal-organic frameworks (MOF(n)) are organic-inorganic hybrid crystalline porous materials that consist of a regular array of positively charged metal ions surrounded by organic 'linker' molecules. The metal ions form nodes that bind the arms of the linkers together to form a repeating, cage-like structure. Moreover, in a chemical structure or molecular graph, edges and vertices are known as bonds and atoms, respectively. Metric dimension being a subsets of atoms with minimum cardinality is used in the substrcturing of the chemical compounds in the molecular structures. Fractional metric dimension is weighted version of metric dimension that associate a numeric value to the identified subset of atoms. In this paper, we have computed the fractional metric dimension of metal organic framework (MOF(n)) for $\mathrm{n} \equiv 0(\bmod ) 2$.

Keywords: metal organic framework, fractional metric dimension, resolving neighborhoods

\section{Introduction and preliminaries}

Metal organic framework (MOF) is a graph that consists of metal atoms. These atoms are linked with the help of organic ligands which acts like a linker, having large pore volume which is known as pours coordination polymer. Therefore, these frameworks led to a new world of remarkable applications. MOF also have large surface area that allow these chemicals compounds to absorb huge quantity of several gases such as carbon

\footnotetext{
* Corresponding author: Muhammad Javaid, Department of Mathematics, School of Science, University of Management and Technology, Lahore, Pakistan, e-mail: javidmath@gamail.com Mohsin Raza and Naeem Saleem: Department of Mathematics, School of Science, University of Management and Technology, Lahore, Pakistan
}

dioxide hydrogen and methane acting as a gas storage chemical compounds. These frameworks are also used for environmental protection and cleaning energy with the help of capturing carbon dioxide. Being small density, high surface, structure edibility and tune able pore functionality. Metal organic frameworks also play an important role in liquid phase separation that is industrial step with critical roles in petrochemical, chemical, nuclear industries and pharmaceutical. MOF is also used in heterogeneous catalyst, drugs delivery and sensing conductivity. Graphical nomenclature of MOF that is represented by a graph $\mathrm{G}$, metal atoms and organic ligands are represented by vertices and edges, respectively (Hasan and Jhung, 2015; Jiao et al., 2019; Liu et al., 2014; Pettinari et al., 2017).

Let $\mathrm{G}$ be the connected graph and distance $u, v \in V(G)$ is represented by $d_{G}(u, v)$, which is length of shortest path from $\mathrm{u}$ to $\mathrm{v}$ in $\mathrm{G}$. Define a resolving set such that, for $u, v \in V(G), R_{G}(u, v)=\{y \in V(G): d(u, y) \neq d(v, y)\}$. A vertex set $W \subseteq V(G)$ is called resolving set of $G$ if $W \cap R_{G}(u, v) \neq \varnothing$ for any two distinct vertices of $\mathrm{G}$. The minimum cardinality among all the resolving set of $G$ is called metric dimension of G. A resloving function $\mathrm{g}$ is called minimal if any function $\mathrm{f}: \mathrm{V}(\mathrm{G}) \rightarrow[0,1]$ such that $\mathrm{f} \leq \mathrm{g}$ and $\mathrm{f}(\mathrm{v}) \neq \mathrm{g}(\mathrm{v})$ for at least one $v \in V$ is not a resolving function of $G$. The fractional metric dimesnison of $G$ is denoted by $\operatorname{dim}_{f}(G)$ and defined as $\operatorname{dim}_{f}(G)=\min \{|g|: g$ is minimal resolving function of $\mathrm{G}\}$, where $|g|=\sum_{v \in V(G)} g(v)$.

Metric dimension has several applications in chemistry, e.g., the substructures of a chemical compound which can be denoted by a set of functional groups. Moreover, in a chemical structure or molecular graph edges and vertices are known as bonds and atoms, respectively. Furthermore, the sub graphs are simply deliberated as substructures and functional groups. Now after altering the position of functional groups, the formed collections of compounds are distinguished as substructures being similar to each other. Later on using the method of traditional view, we can investigate if any two compounds hold the same functional group 


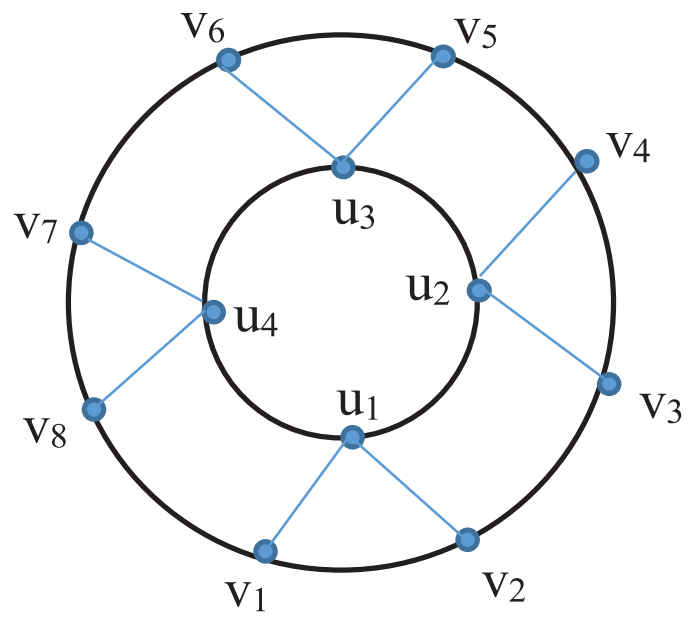

Figure 1: Metal-organic framework (4).

at the same point, while in drugs discovery comparative statement contributes a critical part to determine pharmacology activities related to the feature of compounds.

Metric dimension initially came under investigation through the works of Harary and Melter (1976). Chartrand et al. (2000) used the concept of metric dimension of graphs for the solution integer programming problem (IPPS). Later Currie et al. (2001) used the concept the fractional metric dimension for the better solution of IPPS. Yu et al. (2020) computed the upper bounds of conves polytopes. Mufti et al. (2020) computed the edge metric dimension of barycentric subdivision of Cayley graphs.

Arumugam et al. (2012) introduced many characteristics of the fractional metric dimension for a graph regarding its order. Various results regarding fractional metric dimension of the Cartesian, hierarchical, corona, lexicographic, and comb products of connected graphs, are studied by Feng and Kong (2018), Feng and Wang (2013), and Yi (2015). Feng et al. (2014) find the metric dimension and fractional metric dimension of graphs. Liu et al. (2019) computed the fractional metric dimension of generalized Jahangir graph. Javaid et al. (2020) and Liu et al. (2020) calculated local fractional metric dimensions of different connected graphs.

Let $\operatorname{MOF}(\mathrm{n})$ for $\mathrm{n} \geq 2$ be a metal organic frame works graph with the $\mathrm{V}(\mathrm{MOF}(\mathrm{n}))=\left\{u_{k}: 1 \leq k \leq n\right\}$ U $\left\{v_{l}: 1 \leq l \leq 2 n\right\}$ and $\mathrm{E}(\mathrm{MOF}(\mathrm{n}))=\left\{u_{k} u_{k+1}: 1 \leq k \leq n-1\right\}$ $\mathrm{U}\left\{v_{l} v_{l+1}: 1 \leq l \leq 2 n-1\right\} \cup\left\{u_{n} u_{1}, v_{2 n} v_{1}\right\} \cup\left\{u_{k} v_{l}, u_{k} v_{l+1}\right.$ $1 \leq k \leq n, 1 \leq l \leq 2 n\}$ are vertices and edges set, respectively, as shown in Figure 1 and 2, where $|\mathrm{V}(\mathrm{MOF}(\mathrm{n}))|=3 \mathrm{n}$ and $|\mathrm{E}(\mathrm{MOF}(\mathrm{n}))|=5 \mathrm{n}$.

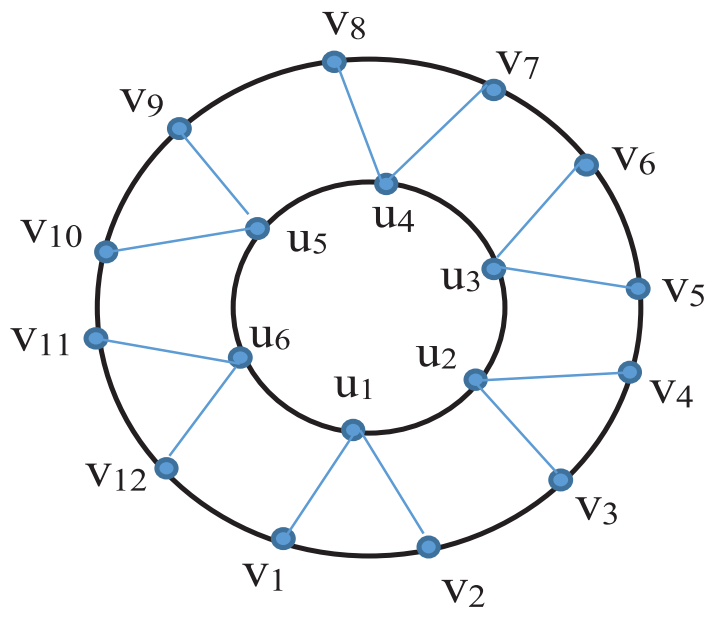

Figure 2: Metal-organic framework (6).

In this paper, we have followed the sequence that first section includes introduction and preliminaries, second section having resolving neighborhood of each possible pair of vertices of $($ MOF $(n))$ for $n=0(\bmod ) 2$. Section 3 consists of main results. The final section of the paper is conclsion.

\section{Resolving neighborhoods of metal organic frame works (MOF)}

Resolving neighborhood of the metal organic frame work $(\mathrm{MOF}(\mathrm{n}))$ for $n \geq 8$ and $\mathrm{n} \equiv 0(\bmod 2)$.

\section{Lemma 1}

Let MOF (n) be the metal organic frame work for $n \geq 8$, $\mathrm{n} \equiv 0(\bmod 2)$. Then, for $1 \leq k \leq n, j=i+1, i \in[2 \mathrm{k}-1]$, and $1 \leq t \leq n$ :

$$
\left|R_{t}\right|=\left|R\left\{v_{i}, v_{j}\right\}\right|=8 .
$$

Moreover, $\bigcup_{t=1}^{n} R_{t}=\left\{v_{m}: 1 \leq m \leq 2 n\right\}$ and $\beta=\left|\bigcup_{t=1}^{n} R_{t}\right|=2 \mathrm{n}$.

Proof: The resolving neighborhoods of metal organic frameworkfor $1 \leq k \leq n, 1 \leq t \leq n, j=i+1, i \in[2 k-1]$ is:

$$
R\left(v_{i}, v_{j}\right)=\left\{\begin{array}{l}
v_{l} \quad \begin{array}{c}
2 k-1 \leq l \leq k-3 \\
2 k-4 \leq l \leq 2 k-2
\end{array}
\end{array}\right.
$$

with,

$$
\left|R_{t}\right|=8
$$

$\bigcup_{t=1}^{n} R_{t}=\left\{v_{p}: 1 \leq p \leq 2 n\right\}$ and $\left|\bigcup_{t=1}^{n} R_{t}\right|=2 n$. 


\section{Lemma 2}

Let MOF (n) be the metal organic frame work for $n \geq 8$ $(\mathrm{n} \equiv 0(\bmod 2)$. Then, for $1 \leq i \leq n, 1 \leq k \leq n-1$, and $1 \leq t \leq n$ :

(a) $\left|R_{t}\right|<\left|R\left(u_{i}, u_{j}\right)\right|$ and $\left|R\left(u_{i}, u_{j}\right) \cap \bigcup_{t=1}^{n} R_{t}\right| \geq\left|R_{t}\right|$, for $j-i \equiv 1(\bmod 2), j \in\{i+k\}$,

(b) $\left|R_{t}\right|<\left|R\left(u_{i}, u_{j}\right)\right|$ and $\left|R\left(u_{i}, u_{j}\right) \cap \bigcup_{t=1}^{n} R_{t}\right| \geq \mid R_{t}$, for $j-i \equiv 0(\bmod 2), j \in\{i+k\}$.

Proof:

(a) The resolving neighborhood for $j-i \equiv 1(\bmod 2)$ :

$$
R\left(u_{i}, u_{j}\right)=\left\{\begin{array}{c}
u_{p}: 1 \leq p \leq n \\
v_{q}: 1 \leq q \leq 2 n
\end{array}\right.
$$

with $\left|R\left(u_{i}, u_{j}\right)\right|=3 n>8=\left|R_{t}\right|$ and $R\left(u_{i}, u_{j}\right) \cap \bigcup_{t=1}^{n} R_{t}=$, $\left\{v_{1}, v_{2}, v_{3, \ldots, \ldots, \ldots, \ldots, \ldots,} v_{2 n},\right\}$

therefore, $\left|R\left(u_{i}, u_{j}\right) \cap \bigcup_{t=1}^{n} R_{t}\right|=2 n>\left|R_{t}\right|$

(b) The resolving neighborhood for $j-i \equiv 0(\bmod 2)$ for $1 \leq i \leq n, 1 \leq t \leq n, j \in\{i+k\}, 1 \leq k \leq n-1$ :

$R\left(u_{i}, u_{j}\right)=$

$\left\{\begin{array}{l}u_{p}: 1 \leq p \leq n: p \neq \frac{i+j}{2}=m \text { and } p \neq \frac{n+i+j}{2}=r, \\ v_{q}: 1 \leq q \leq 2 n: q \neq 2 m, 2 m-1 \text { and } q \neq 2 r, 2 r-1\end{array}\right.$

with $\left|R\left(u_{i}, u_{j}\right)\right|=3 n-6>8=\left|R_{t}\right|$ and $R\left(u_{i}, u_{j}\right) \cap \bigcup^{n} R_{t}=$, $\left\{v_{q}, \& 1 \leq q \leq 2 n: q \neq 2 m, 2 m-1\right.$ and $\left.q \neq 2 r, 2 r-1\right\}$, therefore, $\left|R\left(u_{i}, u_{j}\right) \cap \bigcup_{t=1}^{n} R_{t}\right|=2 n-4>\left|R_{t}\right|$.

\section{Lemma 3}

Let MOF (n) be the metal organic frame work for and $n \geq 8$ and $\mathrm{n} \equiv \mathrm{O}(\bmod 2)$. Then, for $1 \leq i \leq n, 1 \leq t \leq n,\left|R_{t}\right|<$ $\left|R\left(u_{i}, v_{j}\right)\right|$ and $\left|R\left(u_{i}, v_{j}\right) \cap \bigcup_{t=1}^{n} R_{t}\right| \geq\left|R_{t}\right|$ :
(a) $j \in\{2 i-1,2 i\}$
(b) $j \in\{2 i+1\}$
(c) $j \in\{2 i+2\}$
(d) $j \in\{2 i+3,2 i+4\}$
(e) $j \in\{2 i+5\}$
(f) $j \in\{2 i+6\}$
(g) $j \in\{2 i+7\}$
(h) $j \in\{2 i+8\}$
(i) $j \in\{2 i+9\}$
(j) $j \in\{2 i+10\}$
(k) $j \in\{2 i+10+k: 1 \leq k \leq 2 n-22$ where $k=4 y-3,4 y-2$ for $\left.1 \leq y \leq \frac{n}{2}-5\right\}$

(l) $j \in\{2 i+10+k: 1 \leq k \leq 2 n-22$ where $k=4 y-1,4 y$ for $\left.1 \leq y \leq \frac{n}{2}-6\right\}$.

Proof:

(a) The resolving neighborhood for $n \geq 8,1 \leq i \leq n, 1 \leq t \leq n$, and $j \in\{2 i-1,2 i\}$.

$R\left(u_{i}, v_{j}\right)=\left\{\begin{array}{c}u_{p}: 1 \leq p \leq n, \\ v_{q}: 1 \leq q \leq 2 n: q \neq j+1, j+2, j-2, j-3 \\ \text { when } j \in\{2 i-2\} \\ q \neq j+2, j+3, j-1, j-2 \\ \text { when } j \in\{2 i\}\end{array}\right.$ with $\left|R\left(u_{i}, v_{j}\right)\right|=3 n-4>8=\left|R_{t}\right|$ and $R\left(u_{i}, v_{j}\right) \cap \bigcup^{n} R_{t}=$ $\left\{v_{q}: 1 \leq q \leq 2 n\right.$ and $q \neq j+1, j+2, j-2, j-3$ when $j \in\{2 i-2\} q \neq j+2, j+3, j-1, j-2$ when $j \in\{2 i\}$,

therefore, $\left|R\left(u_{i}, v_{j}\right) \cap \bigcup_{t=1}^{n} R_{t}\right|=2 n-4>\left|R_{t}\right|$.

(b) The resolving neighborhood for $n \geq 8,1 \leq i \leq n$, $1 \leq t \leq n$, and $j \in\{2 i+1\}$.

$$
\begin{aligned}
& R\left(u_{i}, v_{j}\right)= \\
& \left\{\begin{array}{l}
u_{p}: 1 \leq p \leq n: p \neq m, \quad j-i \leq m \leq \frac{n}{2} \\
v_{q}: 1 \leq q \leq 2 n: q \neq j-1, q \neq t \text { and } j+3 \leq t \leq \frac{n}{2}+3
\end{array}\right.
\end{aligned}
$$$$
\text { with }\left|R\left(u_{i}, v_{j}\right)\right|=3 n-16>8=\left|R_{t}\right| \text { and }
$$$$
R\left(u_{i}, v_{j}\right) \cap \bigcup_{t=1}^{n} R_{t}=\left\{v_{q}, 1 \leq q \leq 2 n: q \neq j-1, q \neq t\right.
$$
and $\left.j+3 \leq t \leq \frac{n}{2}+3\right\}$,

therefore, $\left|R\left(u_{i}, u_{j}\right) \cap \bigcup_{t=1}^{n} R_{t}\right|=2 n-10>\left|R_{t}\right|$.

(c) The resolving neighborhood for $n \geq 8,1 \leq i \leq n$, $1 \leq t \leq n$, and $j \in\{2 i+2\}$.

$$
\begin{aligned}
& R\left(u_{i}, v_{j}\right)= \\
& \qquad\left\{\begin{array}{l}
u_{p}: 1 \leq p \leq n: p \neq m, \quad j-i-1 \leq m \leq \frac{n}{2} \\
v_{q}: 1 \leq q \leq 2 n: q \neq s \text { and } 2(j-i+1) \leq s \leq \frac{n}{2}+1
\end{array}\right. \\
& \text { with }\left|R\left(u_{i}, v_{j}\right)\right|=3 n-13>8=\left|R_{t}\right| \text { and } \\
& R\left(u_{i}, v_{j}\right) \cap \bigcup_{t=1}^{n} R_{t}=\left\{v_{q}, 1 \leq q \leq 2 n: q \neq s\right. \text { and } \\
& \left.2(j-i+1) \leq s \leq \frac{n}{2}+1\right\},
\end{aligned}
$$

therefore, $\left|R\left(u_{i}, v_{j}\right) \cap \bigcup_{t=1}^{n} R_{t}\right|=2 n-7>\left|R_{t}\right|$. 
(d) The resolving neighborhood for

$n \geq 8,1 \leq i \leq n, 1 \leq t \leq n$, and $j \in\{2 i+3,2 i+4\}$.

$$
R\left(u_{i}, v_{j}\right)=\left\{\begin{array}{l}
u_{p}: 1 \leq p \leq n \\
v_{q}: 1 \leq q \leq 2 n: q \neq j-2
\end{array}\right.
$$

with $\left|R\left(u_{i}, v_{j}\right)\right|=3 n-1>8=\left|R_{t}\right|$ and $R\left(u_{i}, v_{j}\right) \cap \bigcup_{t=1}^{n} R_{t}=\left\{v_{q}: 1 \leq q \leq 2 n: q \neq j-2\right\}$,

therefore, $\left|R\left(u_{i}, v_{j}\right) \cap \bigcup_{t=1}^{n} R_{t}\right|=2 n-1>\left|R_{t}\right|$.

(e) The resolving neighborhood for $n \geq 8,1 \leq i \leq n, 1 \leq t \leq n$ and $j \in\{2 i+5\}$.

$$
R\left(u_{i}, v_{j}\right)=\left\{\begin{array}{l}
u_{p}: 1 \leq p \leq n: p \neq i+2, p \neq \frac{n}{2}+2=s \\
v_{q}: 1 \leq q \leq 2 n: q \neq 2 s, 2 s-1
\end{array}\right.
$$

with $R\left(u_{i}, v_{j}\right)|=3 n-4>8=| R_{t} \mid$ and $R\left(u_{i}, v_{j}\right) \cap \bigcup_{t=1}^{n} R_{t}=\left\{v_{q}, 1 \leq q \leq 2 n: q \neq 2 s, 2 s-1\right\}$, therefore, $\left|R\left(u_{i}, v_{j}\right) \cap \bigcup_{t=1}^{n} R_{t}\right|=2 n-2>\left|R_{t}\right|$.

(f) The resolving neighborhood for $n \geq 8,1 \leq i \leq n, 1 \leq t \leq n$ and $j \in\{2 i+6\}$.

$$
R\left(u_{i}, v_{j}\right)=\left\{\begin{array}{l}
u_{p}: 1 \leq p \leq n: p \neq i+2, p \neq \frac{n}{2}+2=s \\
v_{q}: 1 \leq q \leq 2 n: q \neq 2 s, 2 s-1, \frac{j}{2}+1
\end{array}\right.
$$

with $\left|R\left(u_{i}, v_{j}\right)\right|=3 n-5>8=\left|R_{t}\right|$ and

$$
R\left(u_{i}, v_{j}\right) \cap \bigcup_{t=1}^{n} R_{t}=\left\{v_{q}, 1 \leq q \leq 2 n: q \neq 2 s, 2 s-1, \frac{j}{2}+1\right\} \text {, }
$$

therefore, $\left|R\left(u_{i}, v_{j}\right) \cap \bigcup_{t=1}^{n} R_{t}\right|=2 n-3>\left|R_{t}\right|$.

(g) The resolving neighborhood for $n \geq 8,1 \leq i \leq n, 1 \leq t \leq n$, and $j \in\{2 i+7\}$.

$$
R\left(u_{i}, v_{j}\right)=\left\{\begin{array}{l}
u_{p}: 1 \leq p \leq n: \\
v_{q}: 1 \leq q \leq 2 n: q \neq j-3
\end{array}\right.
$$$$
\text { with }\left|R\left(u_{i}, v_{j}\right)\right|=3 n-1>8=\left|R_{t}\right| \text { and }
$$$$
R\left(u_{i}, v_{j}\right) \cap \bigcup_{t=1}^{n} R_{t}=\left\{v_{q}, 1 \leq q \leq 2 n: q \neq j-3\right\},
$$

therefore, $\left|R\left(u_{i}, v_{j}\right) \cap \bigcup_{t=1}^{n} R_{t}\right|=2 n-3>\left|R_{t}\right|$. (h) The resolving neighborhood for $n \geq 8,1 \leq i \leq n, 1 \leq t \leq n$, and $j \in\{2 i+8\}$.

$$
R\left(u_{i}, v_{j}\right)=\left\{\begin{array}{l}
u_{p}: 1 \leq p \leq n \\
v_{q}: 1 \leq q \leq 2 n
\end{array}\right.
$$

with $\left|R\left(u_{i}, v_{j}\right)\right|=3 n>8=\left|R_{t}\right|$ and

$$
R\left(u_{i}, v_{j}\right) \cap \bigcup_{t=1}^{n} R_{t}=\left\{v_{q}, 1 \leq q \leq 2 n\right\},
$$

therefore, $\left|R\left(u_{i}, v_{j}\right) \cap \bigcup_{t=1}^{n} R_{t}\right|=2 n>\left|R_{t}\right|$.

(i) The resolving neighborhood for $n \geq 8,1 \leq i \leq n, 1 \leq t \leq n$, and $j \in\{2 i+9\}$.

$R\left(u_{i}, v_{j}\right)=\left\{\begin{array}{l}u_{p}: 1 \leq p \leq n: p \neq i+3, p \neq \frac{n+j-5}{2}=s \\ v_{q}: 1 \leq q \leq 2 n: q \neq 2 s, 2 s-1, j-4\end{array}\right.$

with $\left|R\left(u_{i}, v_{j}\right)\right|=3 n-5>8=\left|R_{t}\right|$ and

$$
R\left(u_{i}, v_{j}\right) \cap \bigcup_{t=1}^{n} R_{t}=\left\{v_{q}, 1 \leq q \leq 2 n\right\},
$$

therefore, $\left|R\left(u_{i}, v_{j}\right) \cap \bigcup_{t=1}^{n} R_{t}\right|=2 n-3>\left|R_{t}\right|$.

(j) The resolving neighborhood for $n \geq 8,1 \leq i \leq n, 1 \leq t \leq n$ and $j \in\{2 i+10\}$.

$$
\begin{aligned}
& R\left(u_{i}, v_{j}\right)= \\
& \qquad\left\{\begin{array}{l}
u_{p}: 1 \leq p \leq n: p \neq i+3=s, p \neq \frac{n+j-5}{2}=m \\
v_{q}: 1 \leq q \leq 2 n: q \neq 2 s, 2 s-1,2 m, 2 m-1
\end{array}\right.
\end{aligned}
$$

with $\left|R\left(u_{i}, v_{j}\right)\right|=3 n-6>8=\left|R_{t}\right|$ and

$$
R\left(u_{i}, v_{j}\right) \cap \bigcup_{t=1}^{n} R_{t}=\left\{v_{q}, 1 \leq q \leq 2 n\right\},
$$

therefore, $\left|R\left(u_{i}, v_{j}\right) \cap \bigcup_{t=1}^{n} R_{t}\right|=2 n-4>\left|R_{t}\right|$.

(k) The resolving neighborhood for $n \geq 8,1 \leq i \leq n$, $1 \leq t \leq n, j \in\{2 i+10+k: 1 \leq k \leq 2 n-22$ where $k=4 t-3,4 t-2$ for $\left.1 \leq t \leq \frac{n}{2}-5\right\}$.

$R\left(u_{i}, v_{j}\right)=\left\{\begin{array}{l}u_{p}: 1 \leq p \leq n \\ v_{q}: 1 \leq q \leq 2 n\end{array}\right.$

with $\left|R\left(u_{i}, v_{j}\right)\right|=3 n>8=\left|R_{t}\right|$ and

$R\left(u_{i}, v_{j}\right) \cap \bigcup_{t=1}^{n} R_{t}=\left\{v_{q}, 1 \leq q \leq 2 n\right\}$, 
therefore, $\left|R\left(u_{i}, v_{j}\right) \cap \bigcup_{t=1}^{n} R_{t}\right|=2 n>\left|R_{t}\right|$.

(l) The resolving neighborhood for $n \geq 8,1 \leq i \leq n$,

$1 \leq t \leq n$ and $j \in\{2 i+10+k: 1 \leq k \leq 2 n-22$ where $k=4 t-1,4 t$ for $\left.1 \leq t \leq \frac{n}{2}-6\right\}$,

$$
\begin{aligned}
& R\left(u_{i}, v_{j}\right)= \\
& \left\{\begin{array}{l}
u_{p}: 1 \leq p \leq n: p \neq \frac{i+9}{2}=s, p \neq \frac{n}{2}+i+3=m \\
v_{q}: 1 \leq q \leq 2 n: q \neq 2 s, 2 s-1,2 m, 2 m-1
\end{array}\right.
\end{aligned}
$$

with $\left|R\left(u_{i}, v_{j}\right)\right|=3 n-6>8=\left|R_{t}\right|$ and

$$
R\left(u_{i}, v_{j}\right) \cap \bigcup_{t=1}^{n} R_{t}=\left\{v_{q}, 1 \leq q \leq 2 n: q \neq 2 s,\right.
$$

$2 s-1,2 m, 2 m-1\}$,

therefore, $\left|R\left(u_{i}, v_{j}\right) \cap \bigcup_{t=1}^{n} R_{t}\right|=2 n-4>\left|R_{t}\right|$.

\section{Corollary}

Let MOF (n) be the metal organic frame work for $n \geq 8$ for $\mathrm{n} \equiv 0(\bmod 2)$. Following is the symmetry of cardinalities of resloving neighbourhoods of metal organic framework.

$$
\begin{aligned}
\left.R\left(u_{i}, v_{j}\right)\right) \mid= & \left|R\left(u_{i}, v_{j}\right)\right|, \text { for } 1 \leq i \leq n j \\
& \in\{2 i-1,2 i, \ldots \ldots . .2 i+10\} \text { and } j \\
& \in\{2 i-2,2 i-3, \ldots \ldots . .2 i-13\} .
\end{aligned}
$$

\section{Lemma 4}

Let MOF (n) be the metal organic frame work for $n \geq 8$ for $\mathrm{n} \equiv 0(\bmod 2)$. Then for $1 \leq k \leq n, 1 \leq t \leq n, i \in\{2 k-1\}$.

$\left|R_{t}\right|<\left|R\left(v_{i}, v_{j}\right)\right|$ and $\left|R\left(v_{i}, v_{j}\right) \cap \bigcup_{t=1}^{n} R_{t}\right| \geq\left|R_{t}\right|$,
(a) $j \in\{i+2, i+6\}$
(b) $j \in\{i+3, i+7\}$
(c) $j \in\{i+4, i+8\}$
(d) $j \in\{i+5\}$

Proof:

(a) The resolving neighborhood for $n \geq 8,1 \leq k \leq n$, $1 \leq t \leq n i \in\{2 k-1\}, j \in\{i+2 . i+6\}$.

$$
\begin{aligned}
& R\left(v_{i}, v_{j}\right)=\left\{\begin{array}{l}
u_{p}: 1 \leq p \leq n \\
v_{q}: 1 \leq q \leq 2 n, q \neq \frac{i+j}{2}
\end{array}\right. \\
& \text { with }\left|R\left(v_{i}, v_{j}\right)\right|=3 n-1>8=\left|R_{t}\right| \text { and } \\
& R\left(v_{i}, v_{j}\right) \cap \bigcup_{t=1}^{n} R_{t}=\left\{v_{q}, 1 \leq q \leq 2 n, q \neq \frac{i+j}{2}\right\},
\end{aligned}
$$

therefore, $\left|R\left(v_{i}, v_{j}\right) \cap \bigcup_{t=1}^{n} R_{t}\right|=2 n-1>\left|R_{t}\right|$.

(b) The resolving neighborhood for $n \geq 8,1 \leq k \leq n$, $1 \leq t \leq n, i \in\{2 k-1\}$, and $j \in\{i+3, i+7\}$.

$$
R\left(v_{i}, v_{j}\right)=\left\{\begin{array}{l}
u_{p}: 1 \leq p \leq n \\
v_{q}: 1 \leq q \leq 2 n
\end{array}\right.
$$

with $\left|R\left(v_{i}, v_{j}\right)\right|=3 n>8=\left|R_{t}\right|$ and

$$
R\left(v_{i}, v_{j}\right) \cap \bigcup_{t=1}^{n} R_{t}=\left\{v_{q}, 1 \leq q \leq 2 n\right\},
$$

therefore, $\left|R\left(v_{i}, v_{j}\right) \cap \bigcup_{t=1}^{n} R_{t}\right|=2 n>\left|R_{t}\right|$.

(c) The resolving neighborhood for $n \geq 8,1 \leq k \leq n$, $1 \leq t \leq n, i \in\{2 k-1\}$, and $j \in\{i+4, i+8\}$.

When $j \in\{i+4\}$ :

$R\left(v_{i}, v_{j}\right)=\left\{\begin{array}{l}u_{p}: 1 \leq p \leq n, p \neq \frac{i+j+2}{4}, \frac{n+j-1}{2}=m \\ v_{q}: 1 \leq q \leq 2 n, q \neq \frac{i+j}{3}, 2 m, 2 m-1\end{array}\right.$

When $j \in\{i+8\}$ :

$R\left(v_{i}, v_{j}\right)=\left\{\begin{array}{l}u_{p}: 1 \leq p \leq n, p \neq \frac{i+j+2}{4}, \frac{n+j-1}{2}=s \\ v_{q}: 1 \leq q \leq 2 n, q \neq \frac{i+j}{2}, 2 s, 2 s-1\end{array}\right.$

with $\left|R\left(v_{i}, v_{j}\right)\right|=3 n-5>8=\left|R_{t}\right|$ and

$R\left(v_{i}, v_{j}\right) \cap \bigcup_{t=1}^{n} R_{t}=\left\{1 \leq q \leq 2 n, q \neq \frac{i+j}{3}, 2 m, 2 m-1\right.$

when $j \in\{i+4\} q \neq \frac{i+j}{2}, 2 s, 2 s-1$ when $j \in\{i+8\}$,

therefore, $\left|R\left(v_{i}, v_{j}\right) \cap \bigcup_{t=1}^{n} R_{t}\right|=2 n-3>\left|R_{t}\right|$.

(d) The resolving neighborhood for $n \geq 8,1 \leq k \leq n$, $1 \leq t \leq n, i \in\{2 k-1\}$, and $j \in\{i+5\}$.

$$
\begin{aligned}
& R\left(v_{i}, v_{j}\right)= \\
& \left\{\begin{array}{l}
u_{p}: 1 \leq p \leq n: p \neq \frac{i+j+1}{2}=s, p \neq \frac{n+j-4}{2}=m \\
v_{q}: 1 \leq q \leq 2 n: q \neq 2 s, 2 s-1,2 t, 2 t-1
\end{array}\right. \\
& \text { with }\left|R\left(v_{i}, v_{j}\right)\right|=3 n-6>8=\left|R_{t}\right| \text { and } \\
& R\left(v_{i}, v_{j}\right) \cap \bigcup_{t=1}^{n} R_{t}=\left\{v_{q}, \& 1 \leq q \leq 2 n: q \neq 2 m,\right. \\
& 2 m-1,2 t, 2 t-1\}, \\
& \text { therefore, }\left|R\left(v_{i}, v_{j}\right) \cap \bigcup_{t=1}^{n} R_{t}\right|=2 n-4>\left|R_{t}\right| \text {. }
\end{aligned}
$$




\section{Lemma 5}

Let MOF (n) be the metal organic frame work for $n \geq 8$ and $n \equiv 0(\bmod 2)$. Then for $1 \leq k \leq n, 1 \leq t \leq n$, $i \in\{2 k\} .\left|R_{t}\right|<\left|R\left(v_{i}, v_{j}\right)\right|$, and $\left|R\left(v_{i}, v_{j}\right) \cap \bigcup_{t=1}^{n} R_{t}\right| \geq\left|R_{t}\right|$,

(a) $j \in\{i+1\}$ (b) $j \in\{i+2, i+6\}$ (c) $j \in\{i+4, i+8\}$

(d) $j \in\{i+3, i+7\}$ (e) $j \in\{i+4, i+8\}$.

Proof:

(a) The resolving neighborhood for

$n \geq 8,1 \leq k \leq n, 1 \leq t \leq n i \in\{2 k\}$, and $j \in\{i+1\}:$

$R\left(v_{i}, v_{j}\right)=\left\{\begin{array}{l}u_{p}: 1 \leq p \leq n \\ v_{q}: 1 \leq q \leq 2 n\end{array}\right.$

with $\left|R\left(v_{i}, v_{j}\right)\right|=3 n>8=\left|R_{t}\right|$ and

$R\left(v_{i}, v_{j}\right) \cap \bigcup_{t=1}^{n} R_{t}=\left\{v_{q}, 1 \leq q \leq 2 n\right\}$,

therefore, $\left|R\left(v_{i}, v_{j}\right) \cap \bigcup_{t=1}^{n} R_{t}\right|=2 n>\left|R_{t}\right|$.

(b) The resolving neighborhood for $n \geq 8,1 \leq k \leq n$, $1 \leq t \leq n, i \in\{2 k\}$, and $j \in\{i+2, i+6\}$ :

$$
R\left(v_{i}, v_{j}\right)=\left\{\begin{array}{l}
u_{p}: 1 \leq p \leq n \\
v_{q}: 1 \leq q \leq 2 n, q \neq \frac{i+j}{2}
\end{array}\right.
$$

with $\left|R\left(v_{i}, v_{j}\right)\right|=3 n-1>8=\left|R_{t}\right|$ and

$R\left(v_{i}, v_{j}\right) \cap \bigcup_{t=1}^{n} R_{t}=\left\{v_{q}, 1 \leq q \leq 2 n, q \neq \frac{i+j}{2}\right\}$,

therefore, $\left|R\left(v_{i}, v_{j}\right) \cap \bigcup_{t=1}^{n} R_{t}\right|=2 n-1>\left|R_{t}\right|$.

(c) The resolving neighborhood for $n \geq 8,1 \leq k \leq n$, $1 \leq t \leq n, i \in\{2 k\}, j \in\{i+3 . i+7\}$

$$
\begin{aligned}
& R\left(v_{i}, v_{j}\right)= \\
& \quad\left\{\begin{array}{l}
u_{p}: 1 \leq p \leq n: p \neq \frac{i+j+1}{4}, p \neq \frac{n+j-4}{2}=t \\
v_{q}: 1 \leq q \leq 2 n: q \neq 2 t, 2 t-1
\end{array}\right.
\end{aligned}
$$$$
\text { with }\left|R\left(v_{i}, v_{j}\right)\right|=3 n-4>8=\left|R_{t}\right| \text { and }
$$$$
R\left(v_{i}, v_{j}\right) \cap \bigcup_{t=1}^{n} R_{t}=\left\{v_{q}, 1 \leq q \leq 2 n: q \neq 2 t, 2 t-1\right\} \text {, }
$$

therefore, $\left|R\left(v_{i}, v_{j}\right) \cap \bigcup_{t=1}^{n} R_{t}\right|=2 n-2>\left|R_{t}\right|$. (d) The resolving neighborhood for $n \geq 8,1 \leq k \leq n$, $1 \leq t \leq n, i \in\{2 k\}$, and $j \in\{i+4 . i+8\}$ :

$R\left(v_{i}, v_{j}\right)=\left\{\begin{array}{l}u_{p}: 1 \leq p \leq n: p \neq \frac{i+j}{4}, p \neq \frac{n+j-4}{2}=m \\ v_{q}: 1 \leq q \leq 2 n: q \neq 2 m, 2 m-1, \frac{i+j}{2}\end{array}\right.$

with $\left|R\left(v_{i}, v_{j}\right)\right|=3 n-5>8=\left|R_{t}\right|$ and

$R\left(v_{i}, v_{j}\right) \cap \bigcup_{t=1}^{n} R_{t}=\left\{v_{q}, 1 \leq q \leq 2 n: q \neq 2 m\right.$,

$\left.2 m-1, \frac{i+j}{2}\right\}$,

therefore, $\left|R\left(v_{i}, v_{j}\right) \cap \bigcup_{t=1}^{n} R_{t}\right|=2 n-3>\left|R_{t}\right|$.

(e) The resolving neighborhood for $n \geq 8,1 \leq k \leq n$, $1 \leq t \leq n, i \in\{2 k\}$ and $j \in\{i+5\}$ :

$$
R\left(v_{i}, v_{j}\right)=\left\{\begin{array}{l}
u_{p}: 1 \leq p \leq n \\
v_{q}: 1 \leq q \leq 2 n
\end{array}\right.
$$

with $\left|R\left(v_{i}, v_{j}\right)\right|=3 n>8=\left|R_{t}\right|$ and

$R\left(v_{i}, v_{j}\right) \cap \bigcup_{t=1}^{n} R_{t}=\left\{v_{q}, 1 \leq q \leq 2 n\right\}$,

therefore, $\left|R\left(v_{i}, v_{j}\right) \cap \bigcup_{t=1}^{n} R_{t}\right|=2 n>\left|R_{t}\right|$.

\section{Lemma 6}

Let MOF (n) be the metal organic frame work for $n \geq 8$ and $(\mathrm{n} \equiv 0(\bmod 2)$. Then for $1 \leq i \leq 2 n, 1 \leq t \leq n$ :

(a) $\left|R_{t}\right|<\left|R\left(u_{i}, v_{j}\right)\right|$ and $\left|R\left(u_{i}, v_{j}\right) \cap \bigcup_{t=1}^{n} R_{t}\right| \geq\left|R_{t}\right|$, $j \in\{i+9+k: 1 \leq k \leq 2 n-18$ where $k=4 t-3,4 t-2$ for $\left.1 \leq t \leq \frac{n}{2}-4\right\}$,

(b) $\left|R_{t}\right|<\left|R\left(u_{i}, v_{j}\right)\right|$ and $\left|R\left(u_{i}, v_{j}\right) \cap \bigcup_{t=1}^{n} R_{t}\right| \geq\left|R_{t}\right|$, $j \in\{i+9+k: 1 \leq k \leq 2 n-18$ where $k=4 t-1,4 t$ for $\left.1 \leq t \leq \frac{n}{2}-5\right\}$.

Proof:

(a) The resolving neighborhood for $n \geq 8,1 \leq i \leq 2 n$, $1 \leq t \leq n$, and $j \in\{i+9+k: 1 \leq k \leq 2 n-18$ where $k=4 t-3,4 t-2$ for $\left.1 \leq t \leq \frac{n}{2}-4\right\}$ :

$R\left(u_{i}, v_{j}\right)=\left\{\begin{array}{l}u_{p}: 1 \leq p \leq n \\ v_{q}: 1 \leq q \leq 2 n\end{array}\right.$ 
with $\left|R\left(v_{i}, v_{j}\right)\right|=3 n>8=\left|R_{t}\right|$ and

$$
R\left(v_{i}, v_{j}\right) \cap \bigcup_{t=1}^{n} R_{t}=\left\{v_{q}, 1 \leq q \leq 2 n\right\}
$$

therefore, $\left|R\left(v_{i}, v_{j}\right) \cap \bigcup_{t=1}^{n} R_{t}\right|=2 n>\left|R_{t}\right|$.

(b) The resolving neighborhood for $n \geq 8,1 \leq i \leq 2 n$, $1 \leq t \leq n, j \in\{i+9+k: 1 \leq k \leq 2 n-18$ where $k=4 t-1,4 t$ for $\left.1 \leq t \leq \frac{n}{2}-5\right\}$ :

$$
\begin{aligned}
& R\left(u_{i}, v_{j}\right)= \\
& \left\{\begin{array}{l}
u_{p}: 1 \leq p \leq n: p \neq \frac{i+7}{2}=s, p \neq \frac{n}{2}+i+3=m \\
v_{q}: 1 \leq q \leq 2 n: q \neq 2 m, 2 m-1,2 t, 2 t-1
\end{array}\right.
\end{aligned}
$$

$$
\text { with }\left|R\left(u_{i}, v_{j}\right)\right|=3 n-6 \geq 8=\left|R_{t}\right| \text { and }
$$$$
R\left(u_{i}, v_{j}\right) \cap \bigcup_{t=1}^{n} R_{t}=\left\{v_{q}, 1 \leq q \leq 2 n: q \neq 2 s,\right.
$$$$
2 s-1,2 m, 2 m-1\} \text {, }
$$

therefore, $\left|R\left(u_{i}, v_{j}\right) \cap \bigcup_{t=1}^{n} R_{t}\right|=2 n-4>\left|R_{t}\right|$.

\section{Corollary}

Let MOF (n) be the metal organic frame work for $n \geq 8$ for $n \equiv 0(\bmod 2)$. Following is the symmetry of cardinalities of resloving neighbourhoods of metal organic framework.

(i) $\mid$ for $\left.1 \leq k \leq n, i \in\{2 k-1\} R\left(v_{i}, v_{j}\right)\right)|=| R\left(v_{i}, v_{j}\right) \mid$, where $j \in\{i+2, \ldots \ldots . . i+8\}$ and $j \in\{i-2, \ldots \ldots . . i-8\}$.

(ii) $\left.\mid R\left(v_{i}, v_{j}\right)\right)|=| R\left(v_{i}, v_{j}\right) \mid$, for $1 \leq k \leq n, i \in\{2 k\}$ $j \in\{i+2, \ldots \ldots . i+7\}$ and $j \in\{i-2, \ldots \ldots . i-7\}$.

\section{Fractional metric dimension of metal organic frame work}

In this section fractional metric dimension metal organic frame work MOF $(\mathrm{n})$ for $n \equiv 0(\bmod 2)$ is calculated.

\section{Theorem 1}

The fractional metric dimension of metal organic frame work $\operatorname{MOF}(\mathrm{n})$ for $2 \leq n \leq 6$ isL

$$
\operatorname{dim}_{f}(\operatorname{MOF}(\mathrm{n}))= \begin{cases}\frac{3}{2} & \text { if } n=2 \\ 2 & \text { if } n=4 \\ \frac{3}{2} & \text { if } n=6\end{cases}
$$

Proof:

Case 1: When $\mathrm{n}=2$, then resolving neighbourhoods are as presented in Tables 1 and 2.

For $1 \leq t \leq 12$, each resloving neighbourhood $\left(R_{t}\right)$ having cardinality 4 which is less than all other cardinalities of resolving neighbourhood $R^{m}$ of $M O F(2)$, where, $1 \leq m \leq 3$. Moreover, $\bigcup_{t=1}^{12} R_{t}=V(M O F(2)$ which implies that $\left|\bigcup_{t=1}^{12} R_{t}\right|=10$ and $\left|R^{m} \cap \bigcup_{t=1}^{12} R_{t}\right| \geq\left|R_{t}\right|=8$. Consequently, $\operatorname{dim}_{f}(\operatorname{MOF}(2))<\sum_{1}^{6} \frac{1}{4}=\frac{3}{2}$.

Case 2: When $\mathrm{n}=4$, then resolving neighbourhoods are as presented in Tables 3 and 4.

For $1 \leq t \leq 2$, each resloving neighbourhood $\left(R_{t}\right)$ having cardinality 6 which is less than all othercardinalities of resolving neighbourhood $\left(R^{m}\right)$ of $\operatorname{MOF}(2)$, where, $1 \leq m \leq 64$. Moreover, $\bigcup_{=1}^{2} R_{t}=V(M O F(2)$ which implies that $\left|\bigcup_{t=1}^{2} R_{t}\right|=10$ and $\left|R^{m} \cap \bigcup_{t=1}^{2} R_{t}\right|=2 n-4 \geq\left|R_{t}\right|=8$. Consequently, $\operatorname{dim}_{f}(\operatorname{MOF}(2))<\sum_{t=1}^{12} \frac{1}{6}=2$.

Case 3: When $n=6$, then resolving neighbourhoods are are presented in Tables 5 and 6.

Table 1: Resolving neighbourhoods for $\mathrm{n}=2$

\begin{tabular}{ll}
\hline Resolving sets $\mathbf{n}=\mathbf{2}$ & Elements \\
\hline$R^{1}=R\left(u_{1}, u_{2}\right)$ & $V(\operatorname{MOF}(2))$ \\
$R^{2}=R\left(v_{1}, v_{4}\right)$ & $V(\operatorname{MOF}(2))$ \\
$R^{3}=R\left(v_{2}, v_{3}\right)$ & $V(\operatorname{MOF}(2))$ \\
\hline
\end{tabular}

Table 2: Resolving neighbourhoods with minimum cardnality for $n=2$

\begin{tabular}{ll}
\hline Resolving sets $\mathbf{n}=\mathbf{2}$ & Elements \\
\hline$R_{1}=R\left(u_{1}, v_{1}\right)$ & $V(\operatorname{MOF}(2))-\left\{v_{2}, v_{3}\right\}$ \\
$R_{2}=R\left(u_{1}, v_{2}\right)$ & $V(\operatorname{MOF}(2))-\left\{v_{1}, v_{4}\right\}$ \\
$R_{3}=R\left(u_{1}, v_{3}\right)$ & $V(\operatorname{MOF}(2))-\left\{u_{2}, v_{2}\right\}$ \\
$R_{4}=R\left(u_{1}, v_{4}\right)$ & $V(\operatorname{MOF}(2))-\left\{u_{2}, v_{1}\right\}$ \\
$R_{5}=R\left(u_{2}, v_{1}\right)$ & $V(\operatorname{MOF}(2))-\left\{u_{1}, v_{4}\right\}$ \\
$R_{6}=R\left(u_{2}, v_{2}\right)$ & $V(\operatorname{MOF}(2))-\left\{u_{1}, v_{3}\right\}$ \\
$R_{7}=R\left(u_{2}, v_{3}\right)$ & $V(\operatorname{MOF}(2))-\left\{v_{1}, v_{4}\right\}$ \\
$R_{8}=R\left(u_{2}, v_{4}\right)$ & $V(\operatorname{MOF}(2))-\left\{v_{2}, v_{3}\right\}$ \\
$R_{9}=R\left(v_{1}, v_{2}\right)$ & $V(\operatorname{MOF}(2))-\left\{u_{1}, u_{2}\right\}$ \\
$R_{10}=R\left(v_{1}, v_{3}\right)$ & $V(\operatorname{MOF}(2))-\left\{v_{2}, v_{4}\right\}$ \\
$R_{11}=R\left(v_{2}, v_{4}\right)$ & $V(\operatorname{MOF}(2))-\left\{u_{1}, u_{3}\right\}$ \\
$R_{12}=R\left(v_{3}, v_{4}\right)$ & $V(\operatorname{MOF}(2))-\left\{u_{1}, u_{2}\right\}$ \\
\hline
\end{tabular}


Table 3: Resolving neighbourhoods for $n=4$

\begin{tabular}{|c|c|}
\hline Resolving sets $n=4$ & Elements \\
\hline$R^{1}=R\left(u_{1}, u_{2}\right)$ & $V(\operatorname{MOF}(4))$ \\
\hline$R^{2}=R\left(u_{1}, u_{4}\right)$ & $V(M O F(4))$ \\
\hline$R^{3}=R\left(u_{1}, v_{1}\right)$ & $V(\operatorname{MOF}(4))-\left\{v_{2}, v_{3}, v_{6}, v_{7}\right\}$ \\
\hline$R^{4}=R\left(u_{1}, v_{2}\right)$ & $V(\operatorname{MOF}(4))-\left\{v_{1}, v_{4}, v_{5}, v_{8}\right\}$ \\
\hline$R^{5}=R\left(u_{1}, v_{3}\right)$ & $V(\operatorname{MOF}(4))-\left\{u_{2}, u_{3}, v_{2}, v_{6}\right\}$ \\
\hline$R^{6}=R\left(u_{1}, v_{4}\right)$ & $V(\operatorname{MOF}(4))-\left\{u_{2}, u_{3}\right\}$ \\
\hline$R^{7}=R\left(u_{1}, v_{5}\right)$ & $V(\operatorname{MOF}(4))-\left\{u_{3}, u_{4}, v_{3}, v_{7}\right\}$ \\
\hline$R^{8}=R\left(u_{1}, v_{6}\right)$ & $V(\operatorname{MOF}(4))-\left\{v_{4}, v_{8}\right\}$ \\
\hline$R^{9}=R\left(u_{1}, v_{7}\right)$ & $V(\operatorname{MOF}(4))-\left\{u_{3}, u_{4}\right\}$ \\
\hline$R^{10}=R\left(u_{1}, v_{8}\right)$ & $V(\operatorname{MOF}(4))-\left\{u_{3}, u_{4}, v_{1}, v_{5}\right\}$ \\
\hline$R^{11}=R\left(u_{2}, u_{3}\right)$ & $V(\operatorname{MOF}(4))$ \\
\hline$R^{12}=R\left(u_{2}, v_{1}\right)$ & $V(\operatorname{MOF}(4))-\left\{u_{1}, u_{4}\right\}$ \\
\hline$R^{13}=R\left(u_{2}, v_{2}\right)$ & $V(\operatorname{MOF}(4))-\left\{u_{1}, u_{4}, v_{7}, v_{8}\right\}$ \\
\hline$R^{14}=R\left(u_{2}, v_{3}\right)$ & $V(\operatorname{MOF}(4))-\left\{v_{1}, v_{4}, v_{5}, v_{8}\right\}$ \\
\hline$R^{15}=R\left(u_{2}, v_{4}\right)$ & $V(\operatorname{MOF}(4))-\left\{v_{2}, v_{3}, v_{6}, v_{7}\right\}$ \\
\hline$R^{16}=R\left(u_{2}, v_{5}\right)$ & $V(\operatorname{MOF}(4))-\left\{u_{3}, u_{4}, v_{4}, v_{8}\right\}$ \\
\hline$R^{17}=R\left(u_{2}, v_{6}\right)$ & $V(\operatorname{MOF}(4))-\left\{u_{3}, u_{4}\right\}$ \\
\hline$R^{18}=R\left(u_{2}, v_{7}\right)$ & $V(M O F(4))-\left\{v_{1} v_{5}\right\}$ \\
\hline$R^{19}=R\left(u_{2}, v_{8}\right)$ & $V(\operatorname{MOF}(4))-\left\{v_{2}, v_{6}\right\}$ \\
\hline$R^{20}=R\left(u_{3}, u_{4}\right)$ & $V(\operatorname{MOF}(4))$ \\
\hline$R^{21}=R\left(u_{3}, v_{1}\right)$ & $V(\operatorname{MOF}(4))-\left\{v_{3}, v_{7}\right\}$ \\
\hline$R^{22}=R\left(u_{3}, v_{2}\right)$ & $V(M O F(4))-\left\{v_{3}, v_{8}\right\}$ \\
\hline$R^{23}=R\left(u_{3}, v_{3}\right)$ & $V(\operatorname{MOF}(4))-\left\{u_{1}, u_{2}\right\}$ \\
\hline$R^{24}=R\left(u_{3}, v_{4}\right)$ & $V(\operatorname{MOF}(4))-\left\{u_{1}, u_{2}, v_{1}, v_{5}\right\}$ \\
\hline$R^{25}=R\left(u_{3}, v_{5}\right)$ & $V(\operatorname{MOF}(4))-\left\{v_{2}, v_{3}, v_{6}, v_{7}\right\}$ \\
\hline$R^{26}=R\left(u_{3}, v_{6}\right)$ & $V(\operatorname{MOF}(4))-\left\{v_{1}, v_{4}, v_{5}, v_{8}\right\}$ \\
\hline$R^{27}=R\left(u_{3}, v_{7}\right)$ & $V(\operatorname{MOF}(4))-\left\{u_{1}, u_{4}, v_{2}, v_{6}\right\}$ \\
\hline$R^{28}=R\left(u_{3}, v_{8}\right)$ & $V(\operatorname{MOF}(4))-\left\{u_{1}, u_{4}\right\}$ \\
\hline$R^{29}=R\left(u_{4}, v_{1}\right)$ & $V(\operatorname{MOF}(4))-\left\{u_{1}, u_{2}, v_{4}, v_{8}\right\}$ \\
\hline$R^{30}=R\left(u_{4}, v_{2}\right)$ & $V(\operatorname{MOF}(4))-\left\{u_{1}, u_{2},\right\}$ \\
\hline$R^{31}=R\left(u_{4}, v_{3}\right)$ & $V(M O F(4))-\left\{v_{1}, v_{5}\right\}$ \\
\hline$R^{32}=R\left(u_{4}, v_{4}\right)$ & $V(M O F(4))-\left\{v_{2}, v_{6}\right\}$ \\
\hline$R^{33}=R\left(u_{4}, v_{5}\right)$ & $V(\operatorname{MOF}(4))-\left\{u_{2}, u_{3}\right\}$ \\
\hline$R^{34}=R\left(u_{4}, v_{6}\right)$ & $V(\operatorname{MOF}(4))-\left\{u_{2}, u_{3}, v_{3}, v_{7}\right\}$ \\
\hline$R^{35}=R\left(u_{4}, v_{7}\right)$ & $V(\operatorname{MOF}(4))-\left\{v_{1}, v_{4}, v_{5}, v_{8}\right\}$ \\
\hline$R^{36}=R\left(u_{4}, v_{8}\right)$ & $V(\operatorname{MOF}(4))-\left\{v_{2}, v_{3}, v_{6}, v_{7}\right\}$ \\
\hline$R^{37}=R\left(v_{1}, v_{2}\right)$ & $V(\operatorname{MOF}(4))-\left\{u_{1}, u_{2}, u_{3}, u_{4}\right\}$ \\
\hline$R^{38}=R\left(v_{1}, v_{3}\right)$ & $V(M O F(4))-\left\{v_{2}, v_{6}\right\}$ \\
\hline$R^{39}=R\left(v_{1}, v_{4}\right)$ & $V(M O F(4))$ \\
\hline$R^{40}=R\left(v_{1}, v_{5}\right)$ & $V(\operatorname{MOF}(4))-\left\{u_{2}, u_{4}, v_{6}, v_{7}\right\}$ \\
\hline$R^{41}=R\left(v_{1}, v_{6}\right)$ & $V(\operatorname{MOF}(4))-\left\{u_{2}, u_{4}\right\}$ \\
\hline$R^{42}=R\left(v_{1}, v_{7}\right)$ & $V(\operatorname{MOF}(4))-\left\{v_{4}, v_{8}\right\}$ \\
\hline$R^{43}=R\left(v_{1}, v_{8}\right)$ & $V(\operatorname{MOF}(4))$ \\
\hline$R^{44}=R\left(v_{2}, v_{3}\right)$ & $V(M O F(4))$ \\
\hline$R^{45}=R\left(v_{2}, v_{4}\right)$ & $V(M O F(4))-\left\{v_{3}, v_{7}\right\}$ \\
\hline$R^{46}=R\left(v_{2}, v_{5}\right)$ & $V(\operatorname{MOF}(4))-\left\{u_{2}, u_{4}\right\}$ \\
\hline$R^{47}=R\left(v_{2}, v_{6}\right)$ & $V(\operatorname{MOF}(4))-\left\{u_{2}, u_{4}, v_{4}, v_{8}\right\}$ \\
\hline$R^{48}=R\left(v_{2}, v_{7}\right)$ & $V(\operatorname{MOF}(4))$ \\
\hline$R^{49}=R\left(v_{2}, v_{8}\right)$ & $V(\operatorname{MOF}(4))-\left\{v_{1}, v_{5}\right\}$ \\
\hline$R^{50}=R\left(v_{3}, v_{4}\right)$ & $V(\operatorname{MOF}(4))-\left\{u_{1}, u_{2}, u_{3}, u_{4}\right\}$ \\
\hline$R^{51}=R\left(v_{3}, v_{5}\right)$ & $V(\operatorname{MOF}(4))-\left\{v_{4}, v_{8}\right\}$ \\
\hline$R^{52}=R\left(v_{3}, v_{6}\right)$ & $V(M O F(4))$ \\
\hline$R^{53}=R\left(v_{3}, v_{7}\right)$ & $V(\operatorname{MOF}(4))-\left\{u_{1}, u_{3}, v_{1}, v_{5}\right\}$ \\
\hline$R^{54}=R\left(v_{3}, v_{8}\right)$ & $V(\operatorname{MOF}(4))-\left\{u_{1}, u_{3}\right\}$ \\
\hline$R^{55}=R\left(v_{4}, v_{5}\right)$ & $V(\operatorname{MOF}(4))$ \\
\hline
\end{tabular}

Table 3: (continued)

\begin{tabular}{ll}
\hline Resolving sets $\mathbf{n}=\mathbf{4}$ & Elements \\
\hline$R^{56}=R\left(v_{4}, v_{6}\right)$ & $V(M O F(4))-\left\{v_{1}, v_{5}\right\}$ \\
$R^{57}=R\left(v_{4}, v_{7}\right)$ & $V(M O F(4))-\left\{u_{1}, u_{3}\right\}$ \\
$R^{58}=R\left(v_{4}, v_{8}\right)$ & $V(M O F(4))-\left\{u_{1}, u_{3}, v_{2}, v_{6}\right\}$ \\
$R^{59}=R\left(v_{5}, v_{6}\right)$ & $V(M O F(4))-\left\{u_{1}, u_{2}, u_{3}, u_{4}\right\}$ \\
$R^{60}=R\left(v_{5}, v_{7}\right)$ & $V(M O F(4))-\left\{v_{2}, v_{6}\right\}$ \\
$R^{61}=R\left(v_{5}, v_{8}\right)$ & $V(M O F(4))$ \\
$R^{62}=R\left(v_{6}, v_{7}\right)$ & $V(M O F(4))$ \\
$R^{63}=R\left(v_{6}, v_{8}\right)$ & $V(M O F(4))-\left\{v_{3}, v_{7}\right\}$ \\
$R^{64}=R\left(v_{7}, v_{8}\right)$ & $V(M O F(4))-\left\{u_{1}, u_{2}, u_{3}, u_{4}\right\}$ \\
\hline
\end{tabular}

Table 4: Resolving neighbourhoods with minimum cardnality for $n=4$

\begin{tabular}{ll}
\hline Resolving sets $\mathbf{n}=4$ & Elements \\
\hline$R_{1}=R\left(u_{1}, u_{3}\right)$ & $V(\operatorname{MOF}(4))-\left\{u_{2}, u_{4}, v_{3}, v_{4}, v_{7}, v_{8}\right\}$ \\
$R^{2}=R\left(u_{2}, u_{4}\right)$ & $V(\operatorname{MOF}(4))-\left\{u_{1}, u_{3}, v_{1}, v_{2}, v_{6}, v_{7}\right\}$ \\
\hline
\end{tabular}

For $1 \leq t \leq 6$, each resloving neighbourhood $\left(R_{t}\right)$ having cardinality 8 which is less than all other cardinalities of resolving neighbourhood $\left(R^{m}\right)$ of $M O F(6)$, where, $1 \leq m \leq 147$. Moreover, $\bigcup_{t=1}^{6} R_{t}=V(M O F(6)$ which implies that $\left|\bigcup_{t=1}^{6} R_{t}\right|=10$ and $\left|R^{m} \cap \bigcup_{t=1}^{6} R_{t}\right| \geq\left|R_{t}\right|=8$. Consequently, $\operatorname{dim}_{f}(\operatorname{MOF}(6))<\sum_{t=1}^{12} \frac{1}{8}=\frac{3}{2}$.

\section{Theorem 2}

Let MOF $(\mathrm{n})$ for $n \geq 8$ and $n \equiv 0(\bmod 2)$ be metal organic frame work. Then, $\operatorname{dim}_{f}(\operatorname{MOF}(\mathrm{n}))<\frac{n}{4}$.

Proof:

From Lemma $1-6$ for $n \geq 8$ and $n \equiv 0(\bmod 2), 1 \leq k \leq n$, $1 \leq t \leq n, j=i+1, i \in[2 k-1], \quad\left|R_{t}\right|=\left|R\left\{v_{i}, v_{j}\right\}\right|=8$, $\beta=\left|\bigcup_{t=1}^{n} R_{t}\right|=2 \mathrm{n} .\left|R\left\{v_{i}, v_{j}\right\}\right| \leq|R(x, y)|$ for all $x, y \in$ $V(\mathrm{MOF}(\mathrm{n}))$ for $n \geq 8$ such that $x \neq v_{i}$ and $x \neq v_{j}$, all resloving sets having minimum cardinalities are disjoint. Therefore,

$$
\operatorname{dim}_{f}(\operatorname{MOF}(\mathrm{n}))<\sum_{1}^{\beta} \frac{1}{\left|R_{t}\right|}
$$

where $\beta=2 n$, for $1 \leq k \leq n, 1 \leq t \leq n, j=i+1, i \in[2 k-1]$, we have hence:

$$
\operatorname{dim}_{f}(\operatorname{MOF}(\mathrm{n}))<\frac{n}{4}
$$


Table 5: Resolving neighbourhoods for $n=6$

\begin{tabular}{|c|c|}
\hline Resolving sets $n=6$ & Elements \\
\hline$R^{1}=R\left(u_{1}, u_{2}\right)$ & $V(M O F(6))$ \\
\hline$R^{2}=R\left(u_{1}, u_{3}\right)$ & $V(\operatorname{MOF}(6))-\left\{u_{2}, u_{5}, v_{3}, v_{4}, v_{9}, v_{10}\right\}$ \\
\hline$R^{3}=R\left(u_{1}, u_{4}\right)$ & $V(\operatorname{MOF}(6))$ \\
\hline$R^{4}=R\left(u_{1}, u_{5}\right)$ & $V(\operatorname{MOF}(6))-\left\{u_{3}, u_{6}, v_{5}, v_{6}, v_{11}, v_{12}\right\}$ \\
\hline$R^{5}=R\left(u_{1}, u_{6}\right)$ & $V(M O F(6))$ \\
\hline$R^{6}=R\left(u_{1}, v_{1}\right)$ & $V(M O F(6))-\left\{v_{2}, v_{3}, v_{10}, v_{11}\right\}$ \\
\hline$R^{7}=R\left(u_{1}, v_{2}\right)$ & $V(M O F(6))-\left\{v_{1}, v_{4}, v_{5}, v_{12}\right\}$ \\
\hline$R^{8}=R\left(u_{1}, v_{3}\right)$ & $V(\operatorname{MOF}(6))-\left\{u_{2}, u_{3}, u_{4}, v_{2}, v_{6}, v_{7}, v_{8}\right\}$ \\
\hline$R^{9}=R\left(u_{1}, v_{4}\right)$ & $V(\operatorname{MOF}(6))-\left\{u_{2}, u_{3}, u_{4}, v_{8}\right\}$ \\
\hline$R^{10}=R\left(u_{1}, v_{5}\right)$ & $V(M O F(6))-\left\{u_{3},\right\}$ \\
\hline$R^{11}=R\left(u_{1}, v_{6}\right)$ & $V(\operatorname{MOF}(6))-\left\{v_{4}, v_{9}\right\}$ \\
\hline$R^{12}=R\left(u_{1}, v_{7}\right)$ & $V(M O F(6))-\left\{u_{3}, u_{5}, v_{10}\right\}$ \\
\hline$R^{13}=R\left(u_{1}, v_{8}\right)$ & $V(\operatorname{MOF}(6))-\left\{u_{3}, u_{5}, v_{5}\right\}$ \\
\hline$R^{14}=R\left(u_{1}, v_{9}\right)$ & $V(M O F(6))-\left\{v_{6}, v_{11}\right\}$ \\
\hline$R^{15}=R\left(u_{1}, v_{10}\right)$ & $V(\operatorname{MOF}(6))-\left\{v_{12}\right\}$ \\
\hline$R^{16}=R\left(u_{1}, v_{11}\right)$ & $V(\operatorname{MOF}(6))-\left\{u_{4}, u_{5}, u_{6}, v_{7}\right\}$ \\
\hline$R^{17}=R\left(u_{1}, v_{12}\right)$ & $V(M O F(6))-\left\{u_{4}, u_{5}, u_{6}, v_{1}, v_{7}, v_{8}, v_{9}\right\}$ \\
\hline$R^{18}=R\left(u_{2}, u_{3}\right)$ & $V(M O F(6))$ \\
\hline$R^{19}=R\left(u_{2}, u_{4}\right)$ & $V(\operatorname{MOF}(6))-\left\{u_{3}, u_{6}, v_{5}, v_{6}, v_{11}, v_{12}\right\}$ \\
\hline$R^{20}=R\left(u_{2}, u_{5}\right)$ & $V(\operatorname{MOF}(6))$ \\
\hline$R^{21}=R\left(u_{2}, u_{6}\right)$ & $V(M O F(6))-\left\{u_{1}, u_{4}, v_{1}, v_{2}, v_{7}, v_{8}\right\}$ \\
\hline$R^{22}=R\left(u_{2}, v_{1}\right)$ & $V(M O F(6))-\left\{u_{1}, u_{5}, u_{6}, v_{9}\right\}$ \\
\hline$R^{23}=R\left(u_{2}, v_{2}\right)$ & $V(\operatorname{MOF}(6))-\left\{u_{1}, u_{5}, u_{6}, v_{3}, v_{9}, v_{10}, v_{11}\right\}$ \\
\hline$R^{24}=R\left(u_{2}, v_{3}\right)$ & $V(\operatorname{MOF}(6))-\left\{v_{1}, v_{4}, v_{12}\right\}$ \\
\hline$R^{25}=R\left(u_{2}, v_{4}\right)$ & $V(\operatorname{MOF}(6))-\left\{v_{2}, v_{3}, v_{6}, v_{7}\right\}$ \\
\hline$R^{26}=R\left(u_{2}, v_{5}\right)$ & $V(M O F(6))-\left\{u_{3}, u_{5}, u_{6}, v_{5}, v_{8}, v_{9}, v_{10}\right\}$ \\
\hline$R^{27}=R\left(u_{2}, v_{6}\right)$ & $V(\operatorname{MOF}(6))-\left\{u_{3}, u_{4}, u_{5}, v_{10}\right\}$ \\
\hline$R^{28}=R\left(u_{2}, v_{7}\right)$ & $V(M O F(6))-\left\{v_{5},\right\}$ \\
\hline$R^{29}=R\left(u_{2}, v_{8}\right)$ & $V(\operatorname{MOF}(6))-\left\{v_{6}, v_{11}\right\}$ \\
\hline$R^{30}=R\left(u_{2}, v_{9}\right)$ & $V(M O F(6))-\left\{u_{4}, u_{6}, v_{12}\right\}$ \\
\hline$R^{31}=R\left(u_{2}, u_{10}\right)$ & $V(\operatorname{MOF}(6))-\left\{u_{4}, u_{6}, u_{7}\right\}$ \\
\hline$R^{32}=R\left(u_{2}, v_{11}\right)$ & $V(M O F(6))-\left\{v_{1}, v_{8}\right\}$ \\
\hline$R^{33}=R\left(u_{2}, v_{12}\right)$ & $V(\operatorname{MOF}(6))-\left\{v_{2},\right\}$ \\
\hline$R^{34}=R\left(u_{3}, u_{4}\right)$ & $V(M O F(6))$ \\
\hline$R^{35}=R\left(u_{3}, u_{5}\right)$ & $V(M O F(6))-\left\{u_{1}, u_{4}, v_{1}, v_{2}, v_{7}, v_{8}\right\}$ \\
\hline$R^{36}=R\left(u_{3}, u_{6}\right)$ & $V(\operatorname{MOF}(6))$ \\
\hline$R^{37}=R\left(u_{3}, v_{1}\right)$ & $V(\operatorname{MOF}(6))-\left\{v_{3}, v_{10}\right\}$ \\
\hline$R^{38}=R\left(u_{3}, v_{2}\right)$ & $V(\operatorname{MOF}(6))-\left\{v_{4}\right\}$ \\
\hline$R^{39}=R\left(u_{3}, v_{3}\right)$ & $V(\operatorname{MOF}(6))-\left\{u_{1}, u_{3}, u_{6}, v_{11}\right\}$ \\
\hline$R^{40}=R\left(u_{3}, v_{4}\right)$ & $V(\operatorname{MOF}(6))-\left\{u_{1}, u_{2}, u_{6}, v_{1}, v_{5}, v_{12}\right\}$ \\
\hline$R^{41}=R\left(u_{3}, v_{5}\right)$ & $V(\operatorname{MOF}(6))-\left\{v_{2}, v_{3}, v_{6}, v_{7}\right\}$ \\
\hline$R^{42}=R\left(u_{3}, v_{6}\right)$ & $V(M O F(6))-\left\{v_{4}, v_{5}, v_{8}, v_{9}\right\}$ \\
\hline$R^{43}=R\left(u_{3}, v_{7}\right)$ & $V(M O F(6))-\left\{u_{4}, u_{5}, u_{6}, v_{6}, v_{10}, v_{11}, v_{12}\right\}$ \\
\hline$R^{44}=R\left(u_{3}, v_{8}\right)$ & $V(\operatorname{MOF}(6))-\left\{u_{4}, u_{5}, u_{6}, v_{12},\right\}$ \\
\hline$R^{45}=R\left(u_{3}, v_{9}\right)$ & $V(\operatorname{MOF}(6))-\left\{v_{7}\right\}$ \\
\hline$R^{46}=R\left(u_{3}, v_{10}\right)$ & $V(\operatorname{MOF}(6))-\left\{v_{1}, v_{8}\right\}$ \\
\hline$R^{47}=R\left(u_{3}, v_{11}\right)$ & $V(\operatorname{MOF}(6))-\left\{u_{1}, u_{5}, v_{2}\right\}$ \\
\hline$R^{48}=R\left(u_{3}, v_{12}\right)$ & $V(\operatorname{MOF}(6))-\left\{u_{1}, u_{5}, v_{9}\right\}$ \\
\hline$R^{49}=R\left(u_{4}, u_{5}\right)$ & $V(M O F(6))$ \\
\hline$R^{50}=R\left(u_{4}, u_{6}\right)$ & $V(M O F(6))-\left\{u_{2}, u_{5}, v_{3}, v_{4}, v_{9}, v_{10}\right\}$ \\
\hline$R^{51}=R\left(u_{4}, v_{1}\right)$ & $V(\operatorname{MOF}(6))-\left\{u_{2}, u_{6}, v_{4}\right\}$ \\
\hline$R^{52}=R\left(u_{4}, v_{2}\right)$ & $V(\operatorname{MOF}(6))-\left\{u_{2}, u_{6}, v_{11}\right\}$ \\
\hline
\end{tabular}

Table 5: (continued)

\begin{tabular}{|c|c|}
\hline Resolving sets $n=6$ & Elements \\
\hline$R^{53}=R\left(u_{3}, v_{3}\right)$ & $V(\operatorname{MOF}(6))-\left\{v_{5}, v_{12}\right\}$ \\
\hline$R^{54}=R\left(u_{4}, v_{4}\right)$ & $V(\operatorname{MOF}(6))-\left\{v_{6}\right\}$ \\
\hline$R^{55}=R\left(u_{4}, v_{5}\right)$ & $V(\operatorname{MOF}(6))-\left\{u_{1}, u_{2}, u_{3}, v_{1}\right\}$ \\
\hline$R^{56}=R\left(u_{4}, v_{6}\right)$ & $V(M O F(6))-\left\{u_{1}, u_{2}, u_{3}, v_{1}, v_{2}, v_{3}\right\}$ \\
\hline$R^{57}=R\left(u_{4}, v_{7}\right)$ & $V(\operatorname{MOF}(6))-\left\{v_{4}, v_{5}, v_{8}, v_{9}\right\}$ \\
\hline$R^{58}=R\left(u_{4}, v_{8}\right)$ & $V(M O F(6))-\left\{v_{6}, v_{7}, v_{10}, v_{11}\right\}$ \\
\hline$R^{59}=R\left(u_{4}, v_{9}\right)$ & $V(\operatorname{MOF}(6))-\left\{u_{1}, u_{5}, u_{6}, v_{1}, v_{2}, v_{8}, v_{12}\right\}$ \\
\hline$R^{60}=R\left(u_{4}, v_{10}\right)$ & $V(\operatorname{MOF}(6))-\left\{u_{1}, u_{5}, u_{6}, v_{2}\right\}$ \\
\hline$R^{61}=R\left(u_{4}, v_{11}\right)$ & $V(\operatorname{MOF}(6))-\left\{v_{9}\right\}$ \\
\hline$R^{62}=R\left(u_{4}, v_{12}\right)$ & $V(\operatorname{MOF}(6))-\left\{v_{3}, v_{10}\right\}$ \\
\hline$R^{63}=R\left(u_{5}, u_{6}\right)$ & $V(\operatorname{MOF}(6))$ \\
\hline$R^{64}=R\left(u_{5}, v_{1}\right)$ & $V(\operatorname{MOF}(6))-\left\{v_{3}, v_{10}\right\}$ \\
\hline$R^{65}=R\left(u_{5}, v_{2}\right)$ & $V(\operatorname{MOF}(6))-\left\{u_{5}, u_{12}\right\}$ \\
\hline$R^{66}=R\left(u_{5}, v_{3}\right)$ & $V(\operatorname{MOF}(6))-\left\{u_{1}, v_{6}\right\}$ \\
\hline$R^{67}=R\left(u_{5}, v_{4}\right)$ & $V(\operatorname{MOF}(6))-\left\{u_{1}, v_{1}\right\}$ \\
\hline$R^{68}=R\left(u_{5}, v_{5}\right)$ & $V(\operatorname{MOF}(6))-\left\{v_{2}, v_{7}\right\}$ \\
\hline$R^{69}=R\left(u_{5}, v_{6}\right)$ & $V(\operatorname{MOF}(6))-\left\{v_{8}\right\}$ \\
\hline$R^{70}=R\left(u_{5}, v_{7}\right)$ & $V(\operatorname{MOF}(6))-\left\{v_{3}, u_{2}, u_{3}, u_{4}, v_{3},\right\}$ \\
\hline$R^{71}=R\left(u_{5}, v_{8}\right)$ & $V(\operatorname{MOF}(6))-\left\{u_{2}, u_{3}, u_{4}, v_{3}, v_{4}, v_{5}, v_{9}\right\}$ \\
\hline$R^{72}=R\left(u_{5}, v_{9}\right)$ & $V(\operatorname{MOF}(6))-\left\{v_{6}, v_{7}, v_{10}, v_{11}\right\}$ \\
\hline$R^{73}=R\left(u_{5}, v_{10}\right)$ & $V(\operatorname{MOF}(6))-\left\{v_{1}, v_{8}, v_{9}, v_{12}\right\}$ \\
\hline$R^{74}=R\left(u_{5}, v_{11}\right)$ & $V(\operatorname{MOF}(6))-\left\{u_{1}, u_{2}, u_{6}, v_{2}, v_{3}, v_{4}, v_{10}\right\}$ \\
\hline$R^{75}=R\left(u_{5}, v_{12}\right)$ & $V(\operatorname{MOF}(6))-\left\{u_{1}, u_{2}, u_{6}, v_{1}, v_{3}, v_{4}\right\}$ \\
\hline$R^{76}=R\left(u_{6}, v_{1}\right)$ & $V(\operatorname{MOF}(6))-\left\{u_{1}, u_{2}, u_{3}, v_{4}, v_{5}, v_{6}, v_{12}\right\}$ \\
\hline$R^{77}=R\left(u_{6}, v_{2}\right)$ & $V(\operatorname{MOF}(6))-\left\{u_{1}, u_{2}, u_{3}, v_{6}\right\}$ \\
\hline$R^{78}=R\left(u_{6}, v_{3}\right)$ & $V(M O F(6))-\left\{v_{1}\right\}$ \\
\hline$R^{79}=R\left(u_{6}, v_{4}\right)$ & $V(\operatorname{MOF}(6))-\left\{v_{2}\right\}$ \\
\hline$R^{80}=R\left(u_{6}, v_{5}\right)$ & $V(\operatorname{MOF}(6))-\left\{u_{2}, u_{4}, v_{8}\right\}$ \\
\hline$R^{81}=R\left(u_{6}, v_{6}\right)$ & $V(\operatorname{MOF}(6))-\left\{u_{2}, u_{4}, v_{3}\right\}$ \\
\hline$R^{82}=R\left(u_{6}, v_{7}\right)$ & $V(\mathrm{MOF}(6))-\left\{u_{4}, v_{9}\right\}$ \\
\hline$R^{83}=R\left(u_{6}, v_{8}\right)$ & $V(\operatorname{MOF}(6))-\left\{v_{9}\right\}$ \\
\hline$R^{84}=R\left(u_{6}, v_{9}\right)$ & $V(\operatorname{MOF}(6))-\left\{u_{3}, u_{4}, u_{5}, v_{5}\right\}$ \\
\hline$R^{85}=R\left(u_{6}, v_{10}\right)$ & $V(\operatorname{MOF}(6))-\left\{u_{3}, u_{4}, u_{5}, v_{5}, v_{6}, v_{7}, v_{11}\right\}$ \\
\hline$R^{86}=R\left(u_{6}, v_{11}\right)$ & $V(\operatorname{MOF}(6))-\left\{v_{1}, v_{9}, v_{12}\right\}$ \\
\hline$R^{87}=R\left(u_{6}, v_{12}\right)$ & $V(\operatorname{MOF}(6))-\left\{v_{2}, v_{3}, v_{10}, v_{11}\right\}$ \\
\hline$R^{88}=R\left(v_{1}, v_{3}\right)$ & $V(\operatorname{MOF}(6))-\left\{v_{2}\right\}$ \\
\hline$R^{89}=R\left(v_{1}, v_{4}\right)$ & $V(\operatorname{MOF}(6))$ \\
\hline$R^{90}=R\left(v_{1}, v_{5}\right)$ & $V(\operatorname{MOF}(6))-\left\{u_{2}, u_{5}, v_{3}, v_{9}\right\}$ \\
\hline$R^{91}=R\left(v_{1}, v_{6}\right)$ & $V(\operatorname{MOF}(6))-\left\{u_{1}, u_{5}\right\}$ \\
\hline$R^{92}=R\left(v_{1}, v_{7}\right)$ & $V(\operatorname{MOF}(6))-\left\{v_{5}\right\}$ \\
\hline$R^{93}=R\left(v_{1}, v_{8}\right)$ & $V(\operatorname{MOF}(6))$ \\
\hline$R^{94}=R\left(v_{1}, v_{9}\right)$ & $V(\operatorname{MOF}(6))-\left\{u_{3}, u_{6}, u_{5}, v_{6}, v_{11}\right\}$ \\
\hline$R^{95}=R\left(v_{1}, v_{10}\right)$ & $V(\operatorname{MOF}(6))-\left\{u_{3}, u_{6}, v_{5}, v_{6}\right\}$ \\
\hline$R^{96}=R\left(v_{1}, v_{11}\right)$ & $V(\operatorname{MOF}(6))-\left\{v_{12}\right\}$ \\
\hline$R^{97}=R\left(v_{1}, v_{12}\right)$ & $V(M O F(6))$ \\
\hline$R^{98}=R\left(v_{2}, v_{3}\right)$ & $V(M O F(6))$ \\
\hline$R^{99}=R\left(v_{2}, v_{4}\right)$ & $V(M O F(6))-\left\{v_{3}\right\}$ \\
\hline$R^{100}=R\left(v_{2}, v_{5}\right)$ & $V(M O F(6))-\left\{u_{2}, u_{5}, v_{9}, v_{10}\right\}$ \\
\hline$R^{101}=R\left(v_{2}, v_{6}\right)$ & $V(\operatorname{MOF}(6))-\left\{u_{2}, u_{5}, v_{4}, v_{10}\right\}$ \\
\hline$R^{102}=R\left(v_{2}, v_{7}\right)$ & $V(\operatorname{MOF}(6))-\left\{v_{4}\right\}$ \\
\hline$R^{103}=R\left(v_{2}, v_{8}\right)$ & $V(\operatorname{MOF}(6))-\left\{v_{5}, v_{11}\right\}$ \\
\hline$R^{104}=R\left(v_{2}, v_{9}\right)$ & $V(\operatorname{MOF}(6))-\left\{u_{3}, u_{6},\right\}$ \\
\hline
\end{tabular}


Table 5: (continued)

\begin{tabular}{|c|c|}
\hline Resolving sets $n=6$ & Elements \\
\hline$R^{105}=R\left(v_{2}, v_{10}\right)$ & $V(\operatorname{MOF}(6))-\left\{u_{3}, u_{6}, v_{6}, v_{12}\right\}$ \\
\hline$R^{106}=R\left(v_{2}, v_{11}\right)$ & $V(M O F(6))$ \\
\hline$R^{107}=R\left(v_{2}, v_{12}\right)$ & $V(M O F(6))-\left\{v_{1}\right\}$ \\
\hline$R^{108}=R\left(v_{3}, v_{5}\right)$ & $V(M O F(6))-\left\{v_{4}\right\}$ \\
\hline$R^{109}=R\left(v_{3}, v_{6}\right)$ & $V(M O F(6))$ \\
\hline$R^{110}=R\left(v_{3}, v_{7}\right)$ & $V(M O F(6))-\left\{u_{3}, u_{6}, v_{5}, v_{11}\right\}$ \\
\hline$R^{111}=R\left(v_{3}, v_{8}\right)$ & $V(M O F(6))-\left\{u_{3}, u_{6}, v_{11}, v_{12}\right\}$ \\
\hline$R^{112}=R\left(v_{3}, v_{9}\right)$ & $V(\operatorname{MOF}(6))-\left\{v_{6}, v_{12}\right\}$ \\
\hline$R^{113}=R\left(v_{3}, v_{10}\right)$ & $V(\operatorname{MOF}(6))$ \\
\hline$R^{114}=R\left(v_{3}, v_{11}\right)$ & $V(\operatorname{MOF}(6))-\left\{u_{1}, u_{4}, v_{1}, v_{7}\right\}$ \\
\hline$R^{115}=R\left(v_{3}, v_{12}\right)$ & $V(M O F(6))-\left\{u_{1}, u_{4}, v_{7}, v_{8}\right\}$ \\
\hline$R^{116}=R\left(v_{4}, v_{5}\right)$ & $V(\operatorname{MOF}(6))$ \\
\hline$R^{117}=R\left(v_{4}, v_{6}\right)$ & $V(M O F(6))-\left\{v_{5}\right\}$ \\
\hline$R^{118}=R\left(v_{4}, v_{7}\right)$ & $V(\operatorname{MOF}(6))-\left\{u_{3}, u_{6}, v_{11}, v_{12}\right\}$ \\
\hline$R^{119}=R\left(v_{4}, v_{8}\right)$ & $V(\operatorname{MOF}(6))-\left\{u_{3}, u_{6}, v_{6}, v_{12}\right\}$ \\
\hline$R^{120}=R\left(v_{4}, v_{9}\right)$ & $V(M O F(6))$ \\
\hline$R^{121}=R\left(v_{4}, v_{10}\right)$ & $V(\operatorname{MOF}(6))-\left\{v_{1}, v_{7}\right\}$ \\
\hline$R^{122}=R\left(v_{4}, v_{11}\right)$ & $V(M O F(6))-\left\{u_{4}, u_{6}\right\}$ \\
\hline$R^{123}=R\left(v_{4}, v_{12}\right)$ & $V(\operatorname{MOF}(6))-\left\{u_{1}, u_{4}, v_{2}, v_{8}\right\}$ \\
\hline$R^{124}=R\left(v_{5}, v_{7}\right)$ & $V(\operatorname{MOF}(6))-\left\{v_{6}\right\}$ \\
\hline$R^{125}=R\left(v_{5}, v_{8}\right)$ & $V(M O F(6))$ \\
\hline$R^{126}=R\left(v_{5}, v_{9}\right)$ & $V(\operatorname{MOF}(6))-\left\{u_{1}, u_{4}, v_{1}, v_{7}\right\}$ \\
\hline$R^{127}=R\left(v_{5}, v_{10}\right)$ & $V(\operatorname{MOF}(6))-\left\{u_{1}, u_{4}\right\}$ \\
\hline$R^{128}=R\left(v_{5}, v_{11}\right)$ & $V(\operatorname{MOF}(6))-\left\{v_{2}, v_{8}\right\}$ \\
\hline$R^{129}=R\left(v_{5}, v_{12}\right)$ & $V(\operatorname{MOF}(6))$ \\
\hline$R^{130}=R\left(v_{6}, v_{7}\right)$ & $V(M O F(6))$ \\
\hline$R^{131}=R\left(v_{6}, v_{8}\right)$ & $V(M O F(6))-\left\{v_{7}\right\}$ \\
\hline$R^{132}=R\left(v_{6}, v_{9}\right)$ & $V(\operatorname{MOF}(6))-\left\{u_{1}, u_{4}, v_{1}, v_{2}\right\}$ \\
\hline$R^{133}=R\left(v_{6}, v_{10}\right)$ & $V(M O F(6))-\left\{u_{1}, u_{4}, v_{2}, v_{8}\right\}$ \\
\hline$R^{134}=R\left(v_{6}, v_{11}\right)$ & $V(M O F(6))$ \\
\hline$R^{135}=R\left(v_{6}, v_{12}\right)$ & $V(\operatorname{MOF}(6))-\left\{u_{3}, u_{9}\right\}$ \\
\hline$R^{136}=R\left(v_{7}, v_{9}\right)$ & $V(\operatorname{MOF}(6))-\left\{v_{8}\right\}$ \\
\hline$R^{137}=R\left(v_{7}, v_{10}\right)$ & $V(\operatorname{MOF}(6))$ \\
\hline$R^{138}=R\left(v_{7}, v_{11}\right)$ & $V(M O F(6))-\left\{u_{2}, u_{5}, v_{3}, v_{9}\right\}$ \\
\hline$R^{139}=R\left(v_{7}, v_{12}\right)$ & $V(\operatorname{MOF}(6))-\left\{u_{1}, u_{5},\right\}$ \\
\hline$R^{140}=R\left(v_{8}, v_{9}\right)$ & $V(\operatorname{MOF}(6))$ \\
\hline$R^{141}=R\left(v_{8}, v_{10}\right)$ & $V(\operatorname{MOF}(6))-\left\{v_{9}\right\}$ \\
\hline$R^{142}=R\left(v_{8}, v_{11}\right)$ & $V(\operatorname{MOF}(6))-\left\{u_{2}, u_{5}, v_{3}, v_{4}\right\}$ \\
\hline$R^{143}=R\left(v_{8}, v_{12}\right)$ & $V(M O F(6))-\left\{u_{2}, u_{5}, v_{4}, v_{10}\right\}$ \\
\hline$R^{144}=R\left(v_{9}, v_{11}\right)$ & $V(M O F(6))-\left\{v_{10}\right\}$ \\
\hline$R^{145}=R\left(v_{9}, v_{12}\right)$ & $V(\operatorname{MOF}(6))$ \\
\hline$R^{146}=R\left(v_{10}, v_{11}\right)$ & $V(\operatorname{MOF}(6))$ \\
\hline$R^{147}=R\left(v_{10}, v_{12}\right)$ & $V(M O F(6))-\left\{v_{11}\right\}$ \\
\hline
\end{tabular}

Table 6: Resolving neighbourhoods with minimum cardnality for $n=6$

\begin{tabular}{ll}
\hline Resolving sets $\mathbf{n}=\mathbf{6}$ & Elements \\
\hline$R^{1}=R\left(v_{1}, v_{2}\right)$ & $V(\operatorname{MOF}(6))-\left\{u_{1}, u_{2}, u_{3}, u_{4}, u_{5}, u_{6}, v_{6}, v_{7}, v_{8}, v_{9}\right\}$ \\
$R^{2}=R\left(v_{3}, v_{4}\right)$ & $V(\operatorname{MOF}(6))-\left\{u_{1}, u_{2}, u_{3}, u_{4}, u_{5}, u_{6}, v_{8}, v_{9}, v_{10}, v_{11}\right\}$ \\
$R^{3}=R\left(v_{5}, v_{6}\right)$ & $V(\operatorname{MOF}(6))-\left\{u_{1}, u_{2}, u_{3}, u_{4}, u_{5}, u_{6}, v_{1}, v_{10}, v_{11}, v_{12}\right\}$ \\
$R^{4}=R\left(v_{7}, v_{8}\right)$ & $V(\operatorname{MOF}(6))-\left\{u_{1}, u_{2}, u_{3}, u_{4}, u_{5}, u_{6}, v_{1}, v_{2}, v_{3}, v_{12}\right\}$ \\
$R^{5}=R\left(v_{9}, v_{10}\right)$ & $V(\operatorname{MOF}(6))-\left\{u_{1}, u_{2}, u_{3}, u_{4}, u_{5}, u_{6}, v_{2}, v_{3}, v_{4}, v_{5}\right\}$ \\
$R^{6}=R\left(v_{11}, v_{12}\right)$ & $V(\operatorname{MOF}(6))-\left\{u_{1}, u_{2}, u_{3}, u_{4}, u_{5}, u_{6}, v_{4}, v_{5}, v_{6}, v_{7}\right\}$ \\
\hline
\end{tabular}

Table 7: Fractional metric dimension of MOF(n)

\begin{tabular}{ll}
\hline MOF $(\boldsymbol{n}), \mathbf{n} \equiv \mathbf{0}(\bmod 2)$ & $\begin{array}{l}\text { Upper bounds of fractional } \\
\text { metric dimension }\end{array}$ \\
\hline$n=2$ & $3 / 2$ \\
$n=4$ & 2 \\
$n=6$ & $3 / 2$ \\
$n \geq 8$ & $n / 4$ \\
\hline
\end{tabular}

\section{Conclusions}

In this paper, fractional metric dimension of metal organic frame work $\operatorname{MOF}(\mathrm{n})$ for $n \equiv 0(\bmod 2)$ is computed. The problem is still open to compute the FMD of MOF (n) $n \equiv 1(\bmod 2)$. The details of the obtained results of FMD is given in Table 7.

Research funding: Authors state no funding involved.

Author contributions: Mohsin Raza: writing - original draft, methodology; Muhammad Javaid: resources, formal analysis, visualization, methodology; Naeem Saleem: writing - review and editing, eesources.

Conflict of interest: One of the authors (Muhammad Javaid) is a Guest Editor of the Main Group Metal Chemistry's Special Issue "Topological descriptors of chemical networks: Theoretical studies" in which this article is published.

Data availability statement: The data used to support the finding of this study are includedwithin the article. Additional data can be obtained from the corresponding author upon request.

\section{References}

Arumugam S., Mathew V., The Fractional Metric Dimension of Graphs. Discrete Math. 2012, 312, 184-190.

Chartrand C., Eroh L., Oellermann O.R., Resolvability in Graphs and the Metric Dimension of a Graph. Discrete Appl. Math., 2000, 105, 99-113.

Chu Y.M., Nadeem M.F, Azeem M., Siddiqui M.K, On sharp bounds on partition dimension of convex polytopes. IEEE Access, 2020, 8, 224781-224790.

Currie J., Oellermann O.R, The Metric Dimension and Metric Independence of a Graph. J. Combin. Math. Combin. Comput., 2001, 39, 157-167.

Feng M., Kong Q., On the Fractional Metric Dimension of Corona Product Graphs and Lexicographic Product Graphs. Ars Combinatoria, 2018, 138, 249-260. 
Feng M., Lv B., Wang K., On the Metric Dimension and Fractional Metric Dimension of Graphs. Appl. Anal. Discr. Math., 2014, 170, 55-63.

Feng M., Wang K., On the Metric Dimension and Fractional Metric Dimension of the Hierarchical Product of Graphs. Appl. Anal. Discr. Math., 2013, 7, 302-313.

Harary F., Melter R.A., The Metric Dimension of a Graph. Ars Combinatoria, 1976, 2, 191- 195.

Hasan Z., Jhung S.H., Removal of Hazardous Organics from Water Using Metal Organic Frameworks (MOFs): Plausible Mechanisms for Selective Adsorptions. Hazard. Mat., 2015, 283, 329-339.

Javaid M., Raza M., Kumam P., Liu J.-B., Sharp Bounds of Local Fractional Metric Dimensions of Connected Networks. IEEE, 2020, 8, 172329-172342.

Jiao L., Ruseow J.Y., Scotskinner W., Wang Z.U., Metal Organic Frameworks: Structures and Functional Applications. Mater. Today, 2019, 27, 43-68.
Liu J., Chen L., Cui H., Zhang J., Zhang L., Su C.Y., Applications of Metal Organic Frameworks in Heterogeneous Supramolecular Catalysis. Chem. Soc. Rev., 2014, 43, 6011-6061.

Liu J.-B., Kamaran M., Javaid M., Local Fractional Metric Dimensions of Rotationally Symmetric and Planar Networks. IEEE, 2020, 8, 82404-82420.

Liu J.-B., Kashif A., Rashid T., Javaid M., Fractional Metric Dimension of Generalized Jahangir Graph. Mathematics, 2019, 7, 1-10.

Mufti Z.S., Nadeem M.F., Ahmad A., Ahmad Z., Computation of Edge Metric Dimension of Barycentric Subdivision of Cayley Graphs. Ital. J. Pure Appl. Math., 2020, 44, 714-722.

Pettinari C., Marchetti F., Drozdovc A., Applications of Metal Organic Frameworks. Polym. Int., 2017, 66, 731-744.

Yi E., The Fractional Metric Dimension of Permutation Graphs. Acta Math. Sin., 2015, 31, 367-382. 